\title{
Temporal Profile of Biofilm Formation, Gene Expression and Virulence Analysis in Candida albicans Strains
}

\author{
Patrícia Pimentel de Barros • Rodnei Dennis Rossoni • \\ Felipe De Camargo Ribeiro · Juliana Campos Junqueira • \\ Antonio Olavo Cardoso Jorge
}

Received: 5 April 2016/Accepted: 29 October 2016/Published online: 9 November 2016

(C) Springer Science+Business Media Dordrecht 2016

\begin{abstract}
The characterization of Candida albicans strains with different degrees of virulence became very useful to understand the mechanisms of fungal virulence. Then, the objective of this study was to assess and compare the temporal profiles of biofilms formation, gene expression of $A L S 1, A L S 3, H W P 1, B C R 1$, EFG1, TEC1, SAP5, PLB2 and LIP9 and virulence in Galleria mellonella of C. albicans ATCC18804 and a clinical sample isolated from an HIV-positive patient (CA60). Although the CFU/mL counting was higher in biofilms formed in vitro by ATCC strain, the temporal profile of the analysis of the transcripts of the $C$. albicans strains was elevated to Ca60 compared to strain ATCC, especially in the genes $H W P 1, A L S 3$, SAP 5, PLB2 and $L I P 9$ (up regulation). Ca60 was more pathogenic for $G$. mellonella in the survival assay $(p=0.0394)$ and hemocytes density $(p=0.0349)$, agreeing with upregulated genes that encode the expression of hyphae and hydrolase genes of Ca60. In conclusion, the $C$. albicans strains used in this study differ in the amount of biofilm formation, virulence in vivo and transcriptional profiles of genes analyzed that can change factors associated with colonization, proliferation and survival of $C$. albicans at different
\end{abstract}

P. P. de Barros $(\bowtie) \cdot$ R. D. Rossoni .

F. De Camargo Ribeiro - J. C. Junqueira - A. O. C. Jorge Departament of Biosciences and Oral Diagnosis, Institute of Science and Technology, UNESP - Univ Estadual Paulista, Av. Engenheiro Francisco José Longo 777, São José Dos Campos, SP CEP 12245-000, Brazil e-mail: barrosdnapp@yahoo.com.br niches. SAP5 and HWPl were the genes more expressed in the formation of biofilm in vitro.

Keywords Candida albicans $\cdot$ Biofilms $\cdot$ Gene expression · Galleria mellonella

\section{Introduction}

In all countries worldwide, Candida albicans is the most frequently isolated species, in both superficial and invasive infections and is associated with many cases of mortality. The most affected subjects are HIV-positive individuals and patients undergoing cancer therapy, among others with compromised immune system [1-4].

The major virulence factors include the expression of adhesins and invasins on the cell surface, the morphological transformation capacity of yeast to hyphae, biofilm formation and the secretion of hydrolytic enzymes, among others [2, 5]. Among the virulence factors of $C$. albicans, the capacity to form biofilm in catheters, prostheses (abiotic) and mucosal surfaces (biotic) is one of the most important factors because it facilitates the adhesion, proliferation and spread of this microorganism to other infection sites [6,7].

Genes TECl, BCRI and EFGI are essential for the formation of a mature and stable biofilm resistant to antifungal therapy and to the protection of the immune system, allowing the spread of infections caused by 
this microorganism [7-13]. Target genes, under the influence of $B C R l$, are described as specific hyphae genes, such as $A S L 1, A L S 3$ and $H W P 1$, among others $[11,12]$. The EFGl gene, an important regulatory factor of transcription in biofilm, is involved in the colonization process by $C$. albicans, modifying its expression according to the immune system of the host and conferring to $C$. albicans the capacity to transition from commensal microorganism to opportunistic pathogen status [13]. Several studies [7, 10, 12, 14] have revealed that decreased expression of genes involved in the regulation of the cell transcriptional process can usually contribute, either directly or indirectly, to the adhesion and invasion processes. Changes in the expression levels of genes involved in these functions may lead to a definite compromise of the biofilm structure, contributing to its vulnerability to both antifungal agents and the host's immune system.

Regarding the virulence factors of $C$. albicans, secreted hydrolases also play a key role in pathogenicity [15-19]. Following yeast adhesion to the host cell surface, a morphological transformation occurs and gives rise to new cells, the hyphae. These cells secrete large amounts of hydrolases, which facilitate penetration into the host cell and promote efficient nutrient acquisition [15-17]. Three different classes of these enzymes are expressed by $C$. albicans: proteases $(S A P s)$, lipases (LIPs) and phospholipases (PLBs). The SAP5, LIP9 and PLB2 genes contribute to pathogenicity by means of host tissue degradation, facilitating fungal invasion $[18,19]$.

In vivo studies are fundamental to the study of microbial interactions of pathogenic microorganisms, and the discovery of new antimicrobial agents. An animal model should reproduce the corresponding pathogenesis of infection in humans, including colonization and invasion from a specific route of entry and interaction with the host immune system [20]. In recent decades, a large number of invertebrate models including Galleria mellonella, Caenorhabditis elegans, Drosophila melanogaster and Dictyostelium discoideum, were developed and are being used for experimental study of pathogenicity [21]. These models have provided considerable knowledge in different aspects of microbial infection and have several advantages over mammalian models, such as low cost in the design, ease of use, speed in obtaining results and the possibility of large-scale studies, serving as screening for studies on vertebrates, thus meeting the ethical and legal issues of the principle of the 3Rs (reduction, refinement and replacement) as well as the need to develop alternatives to conventional animals [22-24].

Understanding the pathogenicity mechanisms that C. albicans uses during infections is necessary to the development of new antifungal therapies and methodologies for accurate and rapid diagnosis. The capacity to form biofilm and hydrolase secretion is an essential factor for the maintenance of this microorganism in the environment, which render it able to resist any type of therapy or damage to its structure $[6,25,26]$. Thus, the objective of this study was to assess and compare the temporal profiles of biofilms formation, gene expression of $A L S 1, A L S 3, H W P 1, B C R 1, E F G 1$, TEC1, SAP5, PLB2 and LIP9 and virulence in Galleria mellonella of reference sample $C$. albicans ATCC18804 and a clinical sample isolated from an HIV-positive patient (Ca60).

\section{Materials and Methods}

\section{Samples}

Two C. albicans samples were used in this study, ATCC18804 and a clinical isolate (Ca60) from the oral cavity of an HIV-positive patient. All strains were from the Laboratory of Microbiology of the Institute of Science and Technology São José dos Campos/ UNESP (Univ Estadual Paulista "Julio de Mesquita Filho" - UNESP), and the Ca60 strain was isolated from an oropharyngeal candidiasis lesion of an HIVpositive patient of the Emílio Ribas Infectology Institute (Instituto de Infectologia Emílio Ribas), according to approval of the Ethics Committee (Protocol 051/2009-PH/CEP).This sample was cultured in chromogenic HiCrome Candida medium (Himedia, Mumbai, India), identified by biochemical methods (API20C System- BioMérieux, Paris, France) and confirmed via molecular methods (PCR Multiplex).

\section{Biofilm Formation}

Suspensions of each $C$. albicans strain were prepared from overnight cultures in $5 \mathrm{~mL}$ of yeast-nitrogen base (YNB) broth (Difco Laboratories Inc, Detroit, 
MI, USA) at $37{ }^{\circ} \mathrm{C}$ for $18 \mathrm{~h}$. Then, the cells were centrifuged at $2.000 \times g$ for $10 \mathrm{~min}$, and the supernatant was discarded. The sediment was resuspended in $0.9 \% \mathrm{NaCl}$ and mixed in shaker tubes for $30 \mathrm{~s}$. This cell washing was repeated twice again. Cell densities were adjusted at $10^{7}$ viable cells $/ \mathrm{mL}$ using a hemocytometer.

For the biofilm formation, the methods described by Seneviratne et al. [27] and Costa et al. [28] were used with some changes. Initially, for the biofilms formation, $100 \mu \mathrm{L}$ of the standardized microorganism suspension was pipette into each well of 96-well microtiter plates $\left(\right.$ TPP $^{\circledR}$, Trasadingen, Switzerland). The plates were incubated under stirring at $75 \mathrm{rpm}$ (Quimis, Diadema, São Paulo) at $37{ }^{\circ} \mathrm{C}$ for $90 \mathrm{~min}$ in the initial adhesion stage. After this period, the microorganism supernatant was gently aspirated, and each well was rinsed with $200 \mu \mathrm{L}$ sterile $0.9 \% \mathrm{NaCl}$ saline. Rinsing was repeated twice with sterile $0.9 \%$ $\mathrm{NaCl}$ saline for removal of non-adherent cells. Then, $200 \mu \mathrm{L}$ of YNB broth (Difco Laboratories Inc, Detroit, MI, USA) supplemented with $100 \mathrm{mM}$ of glucose was pipetted, and the plates were incubated at $37^{\circ} \mathrm{C}$ for 12,24 and $48 \mathrm{~h}$ under stirring (Quimis, Diadema, São Paulo). For the 48-h biofilm, the broth was changed after $24 \mathrm{~h}$. For the 0 -h time, the biofilm was removed right after the initial adhesion stage. The experiments were performed in triplicate at different times and with ten replicates per biofilm formation time, totaling 240 assays.

\section{Biofilm Quantification}

Once the formation time of the biofilms had elapsed, the content of the plates was aspirated and rinsed twice with sterile $0.9 \% \mathrm{NaCl}$ saline. Then, $200 \mu \mathrm{L}$ of sterile $0.9 \% \mathrm{NaCl}$ saline was transferred to each well, and the biofilm attached to the plate bottom was broken by homogenization for $30 \mathrm{~s}$ in an ultrasonic homogenizer (Vibra Cell -Sonics \& Materials, Inc. Newtown, USA) with $25 \%$ amplification. A $100 \mu \mathrm{L}$ volume of this inoculum was transferred to $1.5 \mathrm{~mL}$ microtubes containing $900 \mu \mathrm{L}$ sterile $0.9 \% \mathrm{NaCl}$ saline. Using the solution contained in the microtubes, decimal dilutions of the biofilm suspension were performed, from which aliquots of $100 \mu \mathrm{L}$ were seeded into Petri dishes containing chromogenic HiCrome Candida medium (Himedia, Mumbai, India), and the dishes were incubated at $37{ }^{\circ} \mathrm{C}$ for $48 \mathrm{~h}$. After this time, the $\mathrm{CFU} / \mathrm{mL}$ was calculated.

\section{Quantitative RT-PCR}

Total RNA was extracted using the TRIzol $^{\circledR}$ kit (Ambion, Inc., Carlsbad, CA, USA) as recommended by the manufacturer. A volume of $1.0 \mathrm{~mL} \mathrm{TRIzol}{ }^{\circledR}$ was added to a $2.0-\mathrm{mL}$ microtube containing the collected yeast and was incubated at room temperature (RT) for $10 \mathrm{~min}$. Subsequently, $200 \mu \mathrm{L}$ chloroform was added (Sigma-Aldrich, St. Louis, MO, USA), and the microtubes were centrifuged at $12.000 \times g$ for $15 \mathrm{~min}$ at $4{ }^{\circ} \mathrm{C}$. The supernatant was transferred to a new microtube, and $500 \mu \mathrm{L}$ isopropanol (SigmaAldrich, St. Louis, MO, USA) was added. After centrifugation, the sediment obtained was washed with $70 \%$ ethanol (Sigma-Aldrich, St. Louis, MO, USA), centrifuged again and resuspended in $50 \mu \mathrm{L}$ RNA storage buffer (Ambion Inc., Carlsbad, CA, USA). The RNA concentration, purity and quality were checked using a NanoDrop 2000 Spectrophotometer (Thermo Fisher Scientific Inc., Wilmington, DE, USA) and agarose gel electrophoresis (Invitrogen $^{\mathrm{TM}}$, Carlsbad, CA, USA). The gel was stained with ethidium bromide (Invitrogen ${ }^{\mathrm{TM}}$, Carlsbad, CA, USA) and visualized on a transilluminator.

The total extracted RNA $(2 \mu \mathrm{g})$ was treated with DNaseI (Turbo DNase Treatment and Removal Reagents-Ambion, Inc., Carlsbad, CA, USA) and transcribed into complementary DNA (cDNA) using the SuperScript ${ }^{\circledR}$ III First-Strand Synthesis SuperMix Kit for qRT-PCR (Invitrogen ${ }^{\mathrm{TM}}$, Carlsbad, CA, USA) according to the protocols recommended by the manufacturer.

The primers for all genes analyzed in the present study were described and used in the same way as indicated by Nailis et al. [19], Nailis et al. [29] and Hnisz et al. [30], according to Table 1. Their specificity was confirmed for $C$. albicans, and not for the other species of this genus.

Transcribed cDNAs were amplified for the relative quantification of EFG1, TEC1, BCR1, ALS1, ALS3, $H W P 1$, SAP5, LIP9 and PLB2 gene expression levels in relation to the concentration of the reference gene (ACT1). In our study, four reference genes, ACT1, $P M A 1, R I P 1$ and $L S C 2$, were tested in all experimental groups. The results were analyzed at http://www. leonxie.com/referencegene.phpe, and the reference gene chosen was ACTI. 
Table 1 Forward (F) and reverse $(\mathrm{R})$ primers used in real-time PCR for the target and reference genes

\begin{tabular}{lll}
\hline Gene name & Sequence 5' $^{\prime}$ 3' $^{\prime}$ & Reference \\
\hline$A L S 1$ & F- CAACAGGCACCTCAGCATCTAC & Nailis et al. [29] \\
ALS1 & R- CTCCACCAGTAACAGATCCACTAGTAA & Nailis et al. [29] \\
$A L S 3$ & F- CAACTTGGGTTATTGAAACAAAAACA & Nailis et al. [29] \\
$A L S 3$ & R- AGAAACAGAAACCCAAGAACAACCT & Nailis et al. [29] \\
$B C R 1$ & F- AATGCCTGCAGGTTATTTGG & Hnisz et al. [30] \\
$B C R 1$ & R- TTTTAGGTGGTGGTGGCAAT & Hnisz et al. [30] \\
$E F G 1$ & F- CATCACAACCAGGTTCTACAACCAAT & Hnisz et al. [30] \\
$E F G 1$ & R- CTACTATTAGCAGCACCACCC & Hnisz et al. [30] \\
$H W P 1$ & F- GACCGTCTACCTGTGGGACAGT & Nailis et al. [19] \\
$H W P 1$ & R- GCTCAACTTATTGCTATCGCTTATTACA & Nailis et al. [19] \\
LIP9 & F- CGCAAGTTTGAAGTCAGGAAAA & Nailis et al. [19] \\
LIP9 & R- CCCACATTACAACTTTGGCATCT & Nailis et al. [19] \\
$P L B 2$ & F- TGAACCTTTGGGCGACAACT & Nailis et al. [19] \\
$P L B 2$ & R- GCCGCGCTCGTTGTTAA & Nailis et al. [19] \\
$S A P 5$ & F- CCAGCATCTTCCCGCACTT & Nailis et al. [19] \\
$S A P 5$ & R- GCGTAAGAACCGTCACCATATTTAA & Nailis et al. [19] \\
$T E C 1$ & F- TGAGCAACAACAACAACAACCAC & Hnisz et al. [30] \\
$T E C 1$ & R- CTGGGTTGTTGTCATAGTGGCC & Hnisz et al. [30] \\
$A C T 1$ & F- TTTCATCTTCTGTATCAGAGGAACTTAT & Nailis et al. [19] \\
$A C T 1$ & R- ATGGGATGAATCATCAAACAAGAG & Nailis et al. [19] \\
$L S C 2$ & F- CGTCAACATCTTTGGTGGTATTGT & Nailis et al. [19] \\
$L S C 2$ & R- TTGGTGGCAGCAATTAAACCT & Nailis et al. [19] \\
$P M A 1$ & F- TTGCTTATGATAATGCTCCATACGA & Nailis et al. [19] \\
$P M A 1$ & R- TACCCCACAACTTGGCAAGT & Nailis et al. [19] \\
$R I P 1$ & F- TGTCACGGTTCCCATTATGATATTT & Nailis et al. [19] \\
$R I P 1$ & R- TGGAATTTCCAAGTTCAATGGA & Nailis et al. [19] \\
\hline & &
\end{tabular}

The qPCR method assessed the amount of cDNA produced in the exponential phase of the amplification reaction. For the detection system, the SYBR ${ }^{\circledR}$ Green fluorophore was used (Platinum ${ }^{\circledR}$ SYBR $^{\circledR}$ Green qPCR SuperMix-UDG Applied Biosystems, Framingham, MA, USA), which consisted of the following reaction: $10 \mu \mathrm{L}$ Supermix Platinum SYBR Green, 1 $\mu \mathrm{L}$ ROX (reference dye), $300 \mathrm{nM}$ forward primer, $300 \mathrm{nM}$ reverse primer, $3.4 \mu \mathrm{L}$ cDNA solution (diluted 1:5) and 4.6 $\mu \mathrm{L}$ Ultrapure water (Invitrogen $^{\mathrm{TM}}$, Carlsbad, CA, USA). These reactions were assembled in a 96-well plate (Invitrogen ${ }^{\mathrm{TM}}$, Carlsbad, CA, USA) to a final volume of $20 \mu \mathrm{L}$ in each well. For the negative control, all reagents except for cDNA were added to the last wells of the plate, which was then sealed with optical adhesive (Invitrogen ${ }^{\mathrm{TM}}$, Carlsbad, CA, USA). Subsequently, the plate was placed in a StepOnePlus ${ }^{\text {TM }}$ PCR System (Applied
Biosystems, Framingham, MA, USA) and was run on the following cycle: $50{ }^{\circ} \mathrm{C}$ for $2 \mathrm{~min}$, followed by initial denaturation at $95{ }^{\circ} \mathrm{C}$ for $2 \mathrm{~min}$, and 40 cycles at $95{ }^{\circ} \mathrm{C}$ for $15 \mathrm{~s}$ and $60{ }^{\circ} \mathrm{C}$ for $30 \mathrm{~s}$. After the last cycle, the dissociation curve (melt curve) of the samples was analyzed, and the absence of any bimodal curve or abnormal amplification signal was verified every $0.1^{\circ} \mathrm{C}$. The $2^{-\Delta \Delta \mathrm{CT}}$ method was used to analyze relative changes in the gene expression based on the qPCR experiment [31].

\section{G. mellonella Survival}

G. mellonella were infected with $C$. albicans as previously described by Cotter et al. [32] and Junqueira et al. [26]. In brief, G. mellonella caterpillars in the final instar larval stage were stored in the dark and used within 7 days from the date of shipment. 
Sixteen randomly chosen caterpillars $(330 \pm 25 \mathrm{mg})$ were infected for each Candida isolate.

C. albicans inocula were prepared by growing $50 \mathrm{~mL}$ YPD cultures overnight at $30{ }^{\circ} \mathrm{C}$. Cells were pelleted at $1.300 \mathrm{Xg}$ for $10 \mathrm{~min}$ followed by three washes in PBS. Cell densities were determined by hemocytometer count. Candida inocula were confirmed by determining the colony-forming units per milliliter $(\mathrm{CFU} / \mathrm{mL})$ on YPD.

A Hamilton syringe was used to deliver Candida inocula at $10^{6}$ cells/larvae in a $10 \mu \mathrm{L}$ volume into the hemocoel of each larva via the last left proleg. Before injection, the area was cleaned using an alcohol swab. After injection, larvae were incubated in plastic containers $\left(37^{\circ} \mathrm{C}\right)$, and the number of dead $G$. mellonella was scored after $4,8,12$ and $24 \mathrm{~h}$ postinfection. Larvae were considered dead when they displayed no movement in response to touch.

\section{Effects of C. albicans Infection to G. mellonella Hemocyte Density}

Larvae were infected with $C$. albicans by injecting the yeast at the last left pro-leg. Hemocytes were collected from the hemocoel at 4,8 and $12 \mathrm{~h}$ post-injection with C. albicans. Larvae were bled into tubes containing cold sterile insect physiologic saline (IPS) $(150 \mathrm{mM}$ sodium chloride; $5 \mathrm{mM}$ potassium chloride; $100 \mathrm{mM}$ Tris-hydrochloride, $\mathrm{pH} 6.9$ with $10 \mathrm{mM}$ EDTA and $30 \mathrm{mM}$ sodium citrate). The hemocytes were enumerated with the aid of a hemocytometer. We did not differentiate between the six types of hemocytes, and results were averaged from four replicates.

\section{Statistical Analysis}

The data are expressed as the means \pm standard deviations (SD) of the results obtained in each experimental group and each biofilms development time and were analyzed for normal distribution using the Kolmogorov-Smirnov test (SigmaPlot ${ }^{\mathrm{TM}}$ Systat Software, Inc., San Jose, CA, USA).The data from relative quantification of gene expression were analyzed using analysis of variance (ANOVA) and Tukey's test. Killing curves were plotted, and statistical analysis was performed by the log-rank (MantelCox) test. Student's $t$ test was used to compare hemocyte densities and CFU/mL assays. Statistical analysis was performed using the software GraphPad
Prism 5 (GraphPad Software, Inc., California, CA, USA). $P$ value $\leq 0.05$ was considered significant.

\section{Results}

In this study, the biomasses formed in in vitro biofilms from C. albicans ATCC18804 and Ca60 strains (isolated from oropharyngeal candidiasis) using the bottom plate method were quantified by $\mathrm{CFU} / \mathrm{mL}$ count and gene expression (qRT-PCR) related to yeast virulence. In vivo we assessed the pathogenicity of the strains by infecting G. mellonella larvae with Candida strains through the survival curve and hemocytes density.

The ATCC18804 strain produced cell biomasses of $6.197 ; 7.442 ; 7.234$ and $7.315 \log _{10}$, respectively, at 0 , 12,24 and $48 \mathrm{~h}$. A similar result was observed with the Ca60 strain; however, biomass production was lower in relation to the ATCC strain, except at time $0 \mathrm{~h}$ : $6.402 ; 6.92 ; 6.774$ and $6.682 \log _{10}$ of biomass at times $0,12,24$ and $48 \mathrm{~h}$. In the comparison between the two strains, we observed a statistically significant difference for all evaluated times, $0 \mathrm{~h}(p=0.0079), 12 \mathrm{~h}$ $(p=0.0001), 24 \mathrm{~h}(p=0.0001)$ and $48 \mathrm{~h}(p=0.0001)$ as shown in Fig. 1.

All the primers employed in this study were specific for the targeted C. albicans genes and amplified in the expected PCR fragment, which were subjected to agarose gel electrophoresis to confirm the molecular weight (data not shown). The efficiency of PCR amplification was between 95 and $100 \%$ for each primer tested, indicating validation of the primers and the standardization of the qPCR assays. The analysis of the obtained melting curves confirmed the presence of a single peak, demonstrating the specificity of the tested primers.

The expression levels of the adhesion genes ( $A L S 1$, $A L S 3$ and $H W P 1)$, transcriptional regulatory genes (TEC1, BCRl and EFG1), and hydrolase genes (SAP5, $P L B 2$ and $L I P 9$ ) were quantified in the cells of the ATCC18804 and Ca60 strains at 0, 12, 24 and 48 h of biofilms development using qPCR (Fig. 2).

In the ATCC18804 strain, expression levels of the transcriptional genes at time $12 \mathrm{~h}$ for TECl, BCRl and $E F G 1$ were significantly increased compared to the control $(p=0.0001)$; however, at the other times, gradual decreases were observed, with no significant differences. The $A L S 1$ expression levels obtained were 


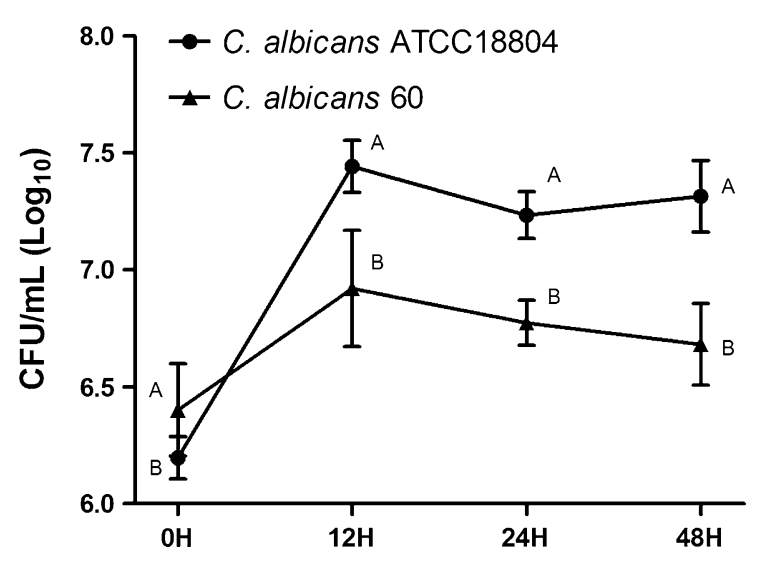

Fig. 1 Temporal quantification in between 0, 12, 24 and $48 \mathrm{~h}$ of biofilms formed by $C$. albicans strains using the viable cell quantification method $(\mathrm{CFU} / \mathrm{mL})$. Values are expressed as the means and standard deviation. Different letters represent significant differences between the ATCC18804 and Ca60 strains at the same time (Student's $t$ test, $p<0.05$ )

low in relation to the control at all times analyzed. ALS3 gene expression was higher at $12 \mathrm{~h}$ but not significant, and gradual and significant decreases were observed at the other times $(p=0.0001)$. The genes related to hydrolases (SAP5, PLB2 and LIP9) and adherence $(H W P 1)$ displayed similar patterns, with gradual increases in the transcript levels over the different biofilm formation times, and a significant value ( $p=0.0001$ ) was observed only for the PLB2 gene at $48 \mathrm{~h}$ (Table 2).

The Ca60 strain exhibited a different gene expression pattern compared to the ATCC strain: The values of the transcript levels of genes ALS3, HWP1, TEC1, $B C R 1$, SAP5, PLB2 and LIP9 were much higher than those observed in the reference strain and significant ( $p=0.0001$ ) compared to the control. In addition, the $H W P 1$ adhesion gene expression level was expressive and significant and increased gradually at all biofilm formation times compared to both the control $(p=0.0001)$ and the reference strain. The expression levels of the PLB2 and LIP9 genes were significantly higher ( $p=0.0001)$ at 12 and $48 \mathrm{~h}$ in relation to both the control and the ATCC strain. However, SAP5 exhibited significantly greater expression at $24 \mathrm{~h}$ $(p=0.0001)$ and decreased expression at 12 and $48 \mathrm{~h}$. Transcriptional genes TECl and EFGl had significantly higher expression levels at $12 \mathrm{~h}$; however, they decreased gradually at $24 \mathrm{~h}$ and $48 \mathrm{~h}$ compared to both the control and the ATCC strain, suggesting that these genes are important in the initial biofilm formation stage (12 h) but not in the mature biofilms (24 and $48 \mathrm{~h}$ ). The $B C R l$ and $A L S 3$ genes exhibited similar expression profiles to each other, with a significant increase observed at $12 \mathrm{~h}$ and gradual and significant decreases at $24 \mathrm{~h}$ and $48 \mathrm{~h}$ $(p=0.0001)$ (Table 3).

Based on these results, we expanded this study for an in vivo model of experimental candidiasis using $G$. mellonella as a host model. Although both strains were lethal to larvae within $24 \mathrm{~h}$ after infection, Ca60 was more pathogenic $(p=0.0394)$ because it leads to death of the larvae more quickly than ATCC strain as shown in Fig. 3. We also compare the pathogenicity of C. albicans to prime the G. mellonella immune response, evaluating changes in the number of available hemocytes. We tested whether each strain can affect hemocity density at 4, 8 and 12 h post-infection. Hemocyte density for the Ca60 was lower at all times evaluated compared to ATCC strain but was statistically significant only in time $12 \mathrm{~h}$ ( $p=0.0349$; Fig. 4), indicating that this strain is more harmful to the larvae.

\section{Discussion}

This study evaluated the CFU/mL of $C$. albicans cells in biofilms, quantified the expression profiles of the ALS1, ALS3, HWP1, BCR1, EFG1, TEC1, SAP5, PLB2 and $L I P 9$ genes that this yeast uses in the development of diseases and compared the pathogenicity of each strain in G. mellonella model through survival curve and hemocyte counting. Biofilm formation is a complex and sequential process that includes several steps: adhesion of yeast cells to a substrate, proliferation of these cells, formation of hyphae on the most superficial layer of the biofilm, production and accumulation of extracellular matrix and, ultimately, dispersion of these cells [28, 33]. Mature biofilms are far more resistant to both antifungal therapy and the host's immunological factors in comparison with planktonic yeast cells [14]. In vitro assays have become an important tool for understanding the composition and properties of $C$. albicans biofilms because they provide accurate and reproducible quantification of the viable cells that make up this complex structure [34].

In the present study, the biofilms formation of $C$. albicans strains was evaluated using viable cell count (CFU/mL) and qRT-PCR methods. First, the CFU/mL 

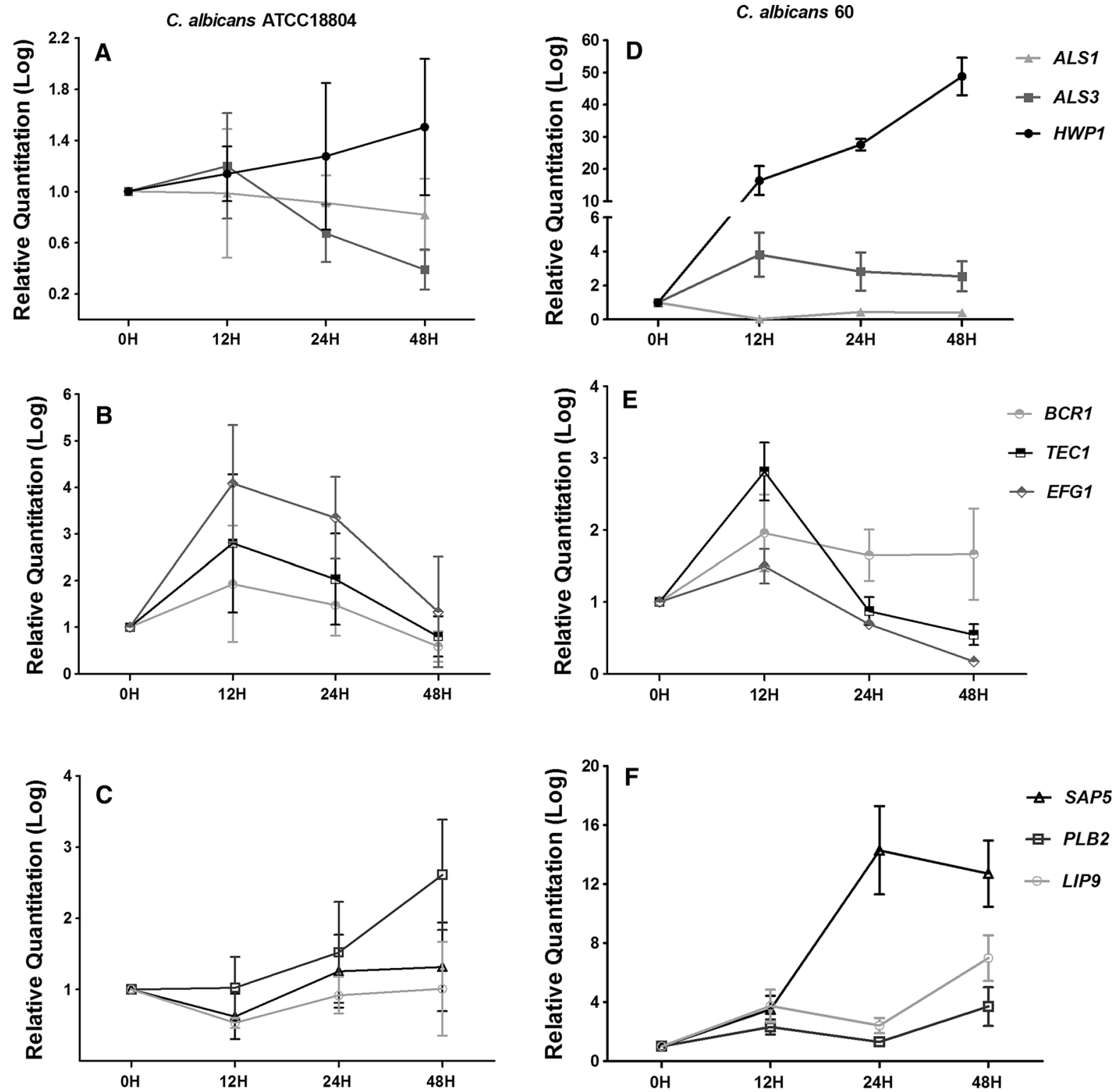

Fig. 2 Relative quantification ( $\log$ ) of the expression levels of adhesion genes (ALS1, ALS3 and HWP1), transcriptional regulatory genes (TEC1, BCR1 and EFG1) and hydrolase genes (SAP5, PLB2 and LIP9) in the cells of ATCC 18804 (a-c) and

Ca60 strains (d-f) at 12, 24 and $48 \mathrm{~h}$ of biofilms formation using qRT-PCR in relation to the control of each strain ( $0 \mathrm{~h}$ time). Values are expressed as the means and standard deviations

counts of the two strains tested were evaluated, indicating that the ATCC18804 strain produced a higher $\mathrm{CFU} / \mathrm{mL}$ compared to the clinical strain. These data suggest that the ATCC18804 strain is more adapted to different in vitro culture methods. Several studies describe the quantification of biofilms formed by $C$. albicans using the CFU/mL method [6, 34-37]. Further, we extended this evaluation to different

biofilm formation times $(0,12,24,48 \mathrm{~h})$, and our results are in agreement with previous reports of a gradual increase in biomass, even at longer times such as $72 \mathrm{~h}$ [19], using the quantitative method of CFU/ $\mathrm{mL}$. The variability observed in the biofilms production kinetics of different $C$. albicans strains when evaluated by various quantification methods maybe due to the origin of the clinical specimen, i.e., the 
Table 2 Values of relative quantification $(\log )$ and standard deviation for all genes analyzed in biofilms of Candida albicans ATCC18804

Control: time immediately after adherence $(0 \mathrm{~h})$

Table 3 Values of relative quantification $(\log )$ and standard deviation for all genes analyzed in biofilms of Candida albicans clinical strain 60

Control: time immediately after adherence $(0 \mathrm{~h})$

Fig. 3 C. albicans (Ca60) is more pathogenic to $G$. mellonella than ATCC strain. Significant differences were observed between C. albicans 60 and C. albicans ATCC 18804 (log-rank test: $p=0.0394$ )

\begin{tabular}{lllll}
\hline Groups & Control & $12 \mathrm{~h}$ & $24 \mathrm{~h}$ & $48 \mathrm{~h}$ \\
\hline ALS1 & 1.0 & $0.98 \pm 0.503$ & $0.91 \pm 0.216$ & $0.82 \pm 0.0279$ \\
ALS3 & 1.0 & $1.20 \pm 0.411$ & $0.67 \pm 0.225$ & $0.39 \pm 0.157$ \\
$H W P 1$ & 1.0 & $1.14 \pm 0.213$ & $1.27 \pm 0.572$ & $1.50 \pm 0.533$ \\
BCR1 & 1.0 & $1.93 \pm 1.244$ & $1.47 \pm 0.652$ & $0.58 \pm 0331$ \\
TEC1 & 1.0 & $2.80 \pm 1.485$ & $2.03 \pm 0.978$ & $0.80 \pm 0.433$ \\
EFG1 & 1.0 & $4.08 \pm 1.258$ & $3.34 \pm 0.880$ & $1.33 \pm 1.184$ \\
SAP5 & 1.0 & $0.62 \pm 0.317$ & $1.25 \pm 0.510$ & $1.32 \pm 0.622$ \\
LIP9 & 1.0 & $0.53 \pm 0.072$ & $0.91 \pm 0.257$ & $1.01 \pm 0.659$ \\
PLB2 & 1.0 & $1.02 \pm 0.436$ & $1.52 \pm 0.709$ & $2.61 \pm 0.776$ \\
\hline
\end{tabular}

\begin{tabular}{llrrr}
\hline Groups & Control & \multicolumn{1}{c}{$12 \mathrm{~h}$} & \multicolumn{1}{c}{$24 \mathrm{~h}$} & \multicolumn{1}{c}{$48 \mathrm{~h}$} \\
\hline ALS1 & 1.0 & $0.03 \pm 0.008$ & $0.45 \pm 0.103$ & $0.41 \pm 0.085$ \\
ALS3 & 1.0 & $3.81 \pm 1.302$ & $2.82 \pm 1.114$ & $2.53 \pm 0.877$ \\
$H W P 1$ & 1.0 & $16.41 \pm 4.471$ & $27.59 \pm 1.812$ & $48.80 \pm 5.831$ \\
BCR1 & 1.0 & $1.95 \pm 0.535$ & $1.64 \pm 0.354$ & $1.66 \pm 0632$ \\
TEC1 & 1.0 & $2.81 \pm 0.402$ & $0.87 \pm 0.194$ & $0.54 \pm 0.145$ \\
EFG1 & 1.0 & $1.49 \pm 0.241$ & $0.69 \pm 0.057$ & $0.17 \pm 0.032$ \\
SAP5 & 1.0 & $3.49 \pm 0.925$ & $14.86 \pm 2.994$ & $12.71 \pm 2.242$ \\
LIP9 & 1.0 & $3.73 \pm 1.101$ & $2.404 \pm 0.514$ & $6.97 \pm 1.551$ \\
PLB2 & 1.0 & $2.30 \pm 0.503$ & $1.30 \pm 0.283$ & $3.70 \pm 1.312$ \\
\hline
\end{tabular}

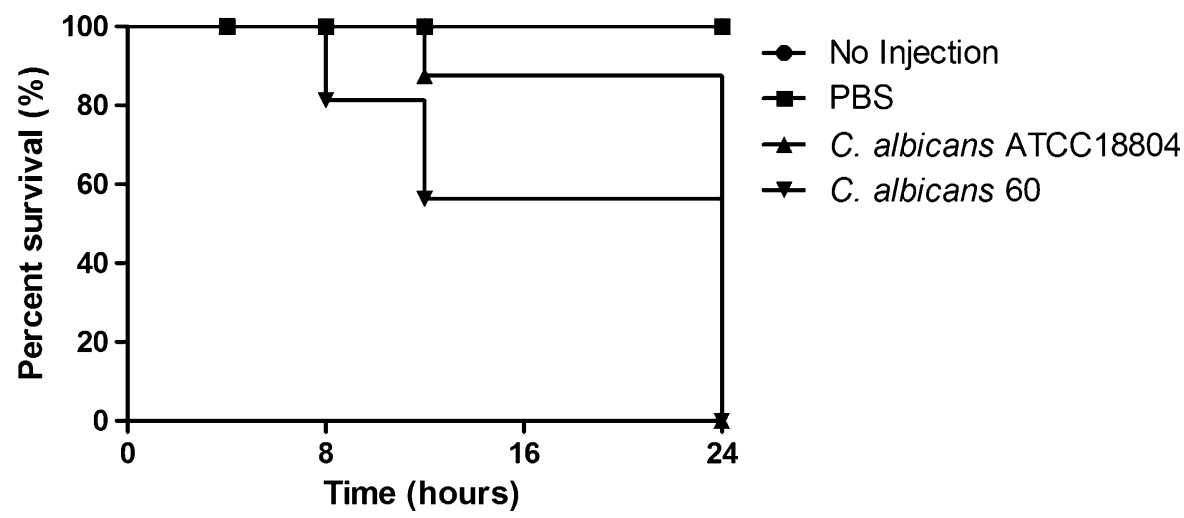

strain isolation site and the factors related to the host's ecological niche, the environment in which factors internal and external to the host directly or indirectly influence the virulence of this yeast.

In an attempt to elucidate the mechanisms that contribute to the differentiation of in vitro biofilms production by strains of the same species, we used relative quantification (qPCR) of genes related to this characteristic that are essential for $C$. albicans pathogenesis. Most studies use relative gene expression to quantify genes involved in the virulence of this yeast against adverse environmental conditions, such as types of nutrients used for growth, production of biofilms in vitro and in vivo, presence or absence of inhibitory substances produced by other microorganisms and sensitivity or resistance to antifungal agents [19, 29, 38-40]. Most studies have evaluated relative gene expression in planktonic cells under the same treatment [26, 29, 35-37]; however, the present study evaluated different genes at different in vitro biofilm 


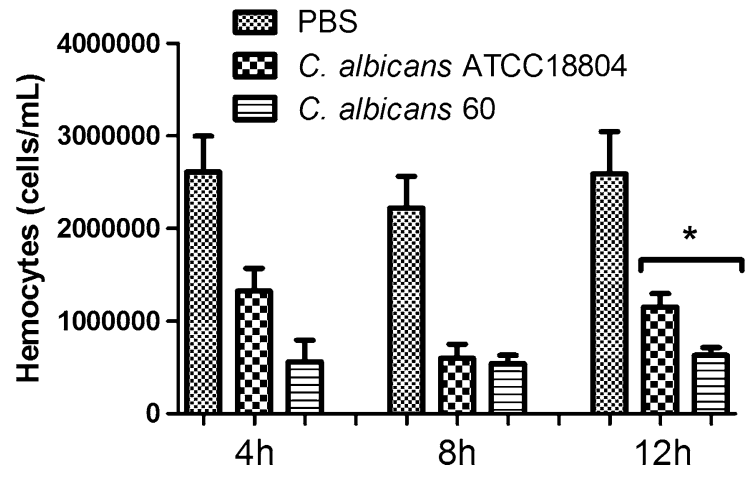

Fig. 4 G. mellonella hemocytes density decreased with the injection of $C$. albicans 60 . The hemolymph was collected to determine the hemocytes densities. Student's $t$ test was used to compare hemocytes densities between the $C$. albicans 60 and $C$. albicans ATCC18804 groups. ${ }^{*} p=0.0349$

formation times, more closely approximating the conditions in which these yeasts are found in the host.

The capacity to form biofilm has been associated with the presence of transcriptional regulatory genes in C. albicans [7]. Among these genes, we can include BCRI, TECl and EFG1 [12-14]. In this study, the ATCC and Ca60 strains differed in the amounts of expression of the TEC1, BCRI and EFG1 genes. In the ATCC strain, the EFGl gene was significantly expressed compared to the control at 12 and $24 \mathrm{~h}$, exhibiting higher values than other biofilms genes. $T E C l$ and $B C R 1$ were positively regulated at $12 \mathrm{~h}$, in accordance with the higher CFU values obtained in this same period. The results for the Ca60 strain were similar in relation to the $12 \mathrm{~h}$ time, where the TECl gene was upregulated compared to $B C R 1$ and $E F G 1$ and gradually decreasing over the formation times. These findings corroborate previous reports, which describe the upregulation of these transcripts in the formation of in vitro and in vivo biofilms [7, 8]. The heterogenic cell activity of the EFGl gene allows colonization by different populations of $C$. albicans against the host's immune system [13]. The BCRl gene positively stimulates gene expression, producing important cell wall proteins involved in the adhesion to both biotic and abiotic surfaces [11, 12]. In the present study, among all evaluated genes, HWP1 exhibited higher upregulation, being gradually expressed over the biofilm formation times in the Ca60 strain, with an expression level 47.3fold higher than the ATCC strain.

Regarding adhesion genes, ALS1 exhibited downregulation in $C$. albicans strains at all analyzed times.
The low expression levels of $A L S 1$ in all strains and different times may be explained by the choice of biofilm development times. According to Yeater et al. [41], ALS1 expression levels are influenced by the culture growth stage, and their conclusions on the degree of expression vary according to comparisons adopted in each analysis; however, there is a consensus in the literature confirming the presence of high levels of $A L S 1$ expression during the biofilms development process [7, 29]. ALS3 was upregulated at $12 \mathrm{~h}$ in both strains; however, Ca60 achieving 3-fold increase compared the ATCC strain at 24 and $48 \mathrm{~h}$. This gene encodes specific hyphal adhesins [7, 9, 19, 29] and is more expressed when we have higher numbers of filaments. This fact may explain why initial and intermediate biofilms express $A L S 3$ more than mature biofilms, regardless of the substrate [19].

The expression of hydrolases SAP5, PLB2 and LIP9 was higher to Ca60 compared to ATCC strain at all times, achieving 11.88 and 9.62-fold increase at 24 and $48 \mathrm{~h}$, respectively. These data are in accordance with previous reports that in the presence of filaments, expression of this gene is stimulated [19, 42].

Nailis et al. [19] compared the amounts of $C$. albicans filaments with the expression $H W P 1$ and all genes of the families $A L S$, SAP, LIP and PLB in biofilms formed on abiotic and biotic surfaces at six biofilm development times $(1,12,24,48,72$ and $144 \mathrm{~h}$ ) by means of qPCR. According to the obtained results, the authors concluded that changes in the environment where the biofilms grow may interfere with its filamentation and gene expression.

In order to elucidate the in vitro results obtained through biofilm formation and gene expression, we investigated the pathogenicity of these strains in $G$. mellonella model. Firstly, we tried to verify if there is a difference in the survival assay between the strains. Ca60 was more pathogenic because it leads to death of the larvae more quickly than ATCC strain. These data are similar with previous studies that evaluated the pathogenicity of $C$. albicans in this model [26, 32, 43, 44].

We also explored alterations of hemocyte density between these two strains and we verified that $\mathrm{Ca} 60$ was capable to reduce the hemocyte density at all times evaluated compared to ATCC strain but was statistically significant only in time $12 \mathrm{~h}$. The density of circulating hemocyte in the hemolymph of $G$. mellonella may be indicative of infection with low 
hemocyte intensity associate with infection and high density indicating insect health [45]. These data agree with our in vitro results that showed increased transcriptional profile of strain Ca60 for genes related to hydrolases (SAP5, PLB2 and LIP9) and filamentation $(H W P 1)$ that are essential for the colonization and invasion of yeast in the host tissue.

More complex studies involving several types of clinical specimens isolated from different sites colonized with $C$. albicans are needed to understand and associate different gene expression profiles with pathogenicity factors to seek new treatment options, in addition to providing knowledge at the molecular level of the effects of virulence factors insusceptible hosts. In conclusion, the C. albicans strains used in this study differ in the amount of biofilm formation, virulence in vivo and transcriptional profiles of genes analyzed that can change factors associated with colonization, proliferation and survival of $C$. albicans at different niches. SAP5 and HWP1 were the genes more expressed in the formation of biofilm in vitro.

Acknowledgements We acknowledge the São Paulo Council of Research-FAPESP, Brazil (Grants 2011/15194-0, 2012/ 15250-0 and 2012/02184-9) for supporting this research.

\section{Compliance with Ethical Standards}

Conflict of interest The authors declare that they have no conflict of interest.

\section{References}

1. Colombo AL, Guimarães T, Camargo LFA, et al. Brazilian guidelines for the management of candidiasis: a joint meeting report of three medical societies. Braz J Infect Dis. 2013;17:283-312.

2. Mayer LF, Wilson D, Hube B. Candida albicans pathogenicity mechanisms. Virulence. 2013;4:119-28.

3. Agwu E, Ihongbe JC, McManus BA, et al. Distribution of yeast species associated with oral lesions in HIV-infected patients in Southwest Uganda. Med Mycol. 2012;50:276-80.

4. Eggimann P, Garbino J, Pittet D. Epidemiology of Candida species infections in critically non-immunosuppressed patients. Lancet Infect Dis. 2003;3:685-702.

5. Hube B. From comensal to pathogen: stage-and tissuespecific gene expression of Candida albicans. Curr Opin Microbiol. 2004; 7:336-41.

6. Hasan F, Xess I, Wang X, Jain N, Fries BC. Biofilm formation in clinical Candida isolates and its association with virulence. Microbes Infect. 2009;11:753-61.

7. Finkel JS, Mitchell AP. Genetic control of Candida albicans biofilm development. Nat Rev Microbiol. 2011;9:109-18.
8. Fox PE, Nobile JC. A sticky situation: untangling the transcriptional network controlling biofilm development in Candida albicans. Transcription. 2012;3:315-22.

9. Nobile CJ, Mitchell AP. Regulation of cell surface genes and biofilm formation by the C. albicans transcription factor Bcr1p. Curr Biol. 2005;15:1150-5.

10. Nobile CJ, Mitchell AP. Genetics and genomics of Candida albicans biofilm formation. Cell Microbiol. 2006;8: 1382-91.

11. Nobile CJ, Andes DR, Nett JE, et al. Critical role of Bcr1dependent adhesins in C. albicans biofilm formation in vitro and in vivo. PLoS Pathog. 2006;2:e63.

12. Nobile CJ, Fox EP, Nett JE, et al. A recently evolved transcriptional network controls biofilm development in Candida albicans. Cell. 2012;148:126-38.

13. Pierce VJ, Kumamoto AC. Variation in Candida albicans EFG1 expression enables host-dependent changes in colonizing fungal populations. MBio. 2012;3:e00117-12.

14. Fanning S, Xu W, Solis N, Woolford CA, et al. Divergent targets of Candida albicans biofilm regulator Bcr1 in vitro and in vivo. Eukar Cell. 2012;11:896-904.

15. Naglik JR, Rodgers CA, Shirlaw PJ, et al. Differential expression of Candida albicans secreted aspartyl proteinase and phospholipase B genes in human correlates with active oral and vaginal infections. J Infect Dis. 2003;188:469-79.

16. Naglik JR, Challacombe SJ, Hube B. Candida albicans aspartyl proteinases in virulence and pathogenesis. Microbiol Mol Biol. 2003;67:400-28.

17. Naglik JR, Albrecht A, Bader O, Hube B. Candida albicans proteinases and host/pathogen interactions. Cell Microbiol. 2004;6:915-26.

18. Naglik JR, Moyes D, Makwana J, et al. Quantitative expression of the Candida albicans secreted aspartyl proteinase gene family in human oral and vaginal candidiasis. Microbiology. 2008;154:3266-80.

19. Nailis H, Kucharíková S, Řičicová M, et al. Real- time PCR expression profiling of genes encoding potential virulence factors in Candida albicans biofilms: identification of model-dependent and -independent gene expression. BMC Microbiol. 2010;10:114.

20. Chamilos G, Lionakis MS, Lewis RE, Kontoyiannis DP. Role of mini-host models in the study of medically important fungi. Lancet Infect Dis. 2007;7:42-55.

21. Arvantis M, Glavis-Bloom J, Mylonakis E. C.elegans for anti-infective discovery. Curr Opin Pharmacol. 2013;13: 769-74.

22. Fedhila S, Buisson C, Dussurget O, et al. Comparative analysis of the virulence of invertebrate and mammalian pathogenic bacteria in the oral insect infection model Galleria mellonella. J Invertebr Pathol. 2010;103:24-9.

23. Chibebe Junior J, Fuchs BB, Sabino CP, et al. Photodynamic and antibiotic therapy impair the pathogenesis of Enterococcus faecium in a whole animal insect model. PLoS ONE. 2013;8(2):e55926.

24. Chibebe Junior J, Sabino CP, Tan X, et al. Selective photoinactivation of Candida albicans in the non-vertebrate host infection model Galleria mellonella. BMC Microbiol. 2013;13:217.

25. Fanning S, Mitchell AP. Fungal biofilms. PLoS Pathog. 2012;8:e1002585. 
26. Junqueira JC, Fuchs BB, Muhammed M, et al. Oral Candida albicans from HIV-positive individuals have similar in vitro biofilm-forming ability and pathogenicity as invasive Candida isolates. BMC Microbiol. 2011;4:247.

27. Seneviratne CJ, Silva WJ, Jin LJ, et al. Architectural analysis, viability assessment and growth kinetics of Candida albicans and Candida glabrata biofilms. Arch Oral Biol. 2009;54:1052-60.

28. Costa ACBP, Pereira AC, Freire F, et al. Methods for obtaining reliable and reproducible results in studies of Candida biofilms formed in vitro. Mycoses. 2013;56: 614-22.

29. Nailis H, Coenye T, Van Nieuwerburgh F, et al. Development and evaluation of different normalization strategies for gene expression studies in Candida albicans biofilms by real- time PCR. BMC Mol Biol. 2006;4:7-25.

30. Hnisz D, Bardet AF, Nobile CJ, et al. A Histone deacetylase adjusts transcription kinetics at coding sequences during Candida albicans morphogenesis. PLoS Genet. 2012;8: e1003118.

31. Livak KJ, Schmittgen TD. Analysis of relative gene expression data using real-time quantitative PCR and the 2(Delta Delta C(T)) Method. Methods. 2001;25:402-8.

32. Cotter G, Doyle S, Kavanagh K. Development of an insect model for the in vivo pathogenicity testing of yeasts. FEMS Immunol Med Microbiol. 2000;27:163-9.

33. Kumamoto CA. Candida biofilms. Curr Opin Microbiol. 2002;5:608-11.

34. Taff HT, Nett JE, Andes DR. Comparative analysis of Candida biofilm quantitation assays. Med Mycol. 2012;50:214-8.

35. Ramage G, VandeWalle K, Wickes LB, López RJC. Characteristics of biofilm formation by Candida albicans. Rev Iberoam Micol. 2001;18:163-70.
36. Ramage G, Saville SP, Thomas DP, Lopez-Ribot JL. Candida biofilms: an update. Eukaryot Cell. 2005;4:633-8.

37. Sánchez-Vargas LO, Estrada-Barraza D, Pozos-Guillen AJ, Rivas-Caceres R. Biofilm formation by oral clinical isolates of Candida species. Arch Oral Biol. 2013;58:1318-26.

38. Ding X, Liu Z, Su J, Yan D. Human serum inhibits adhesion and biofilm formation in Candida albicans. BMC Microbiol. 2014;14:80.

39. Buu LM, Chen YC. Impact of glucose levels on expression of hypha-associated secreted aspartyl proteinases in Candida albicans. J Biomed Sci. 2014;21:22.

40. Semlali A, Killer K, Alanazi H, Chmielewski W, et al. Cigarette smoke condensate increases $C$. albicans adhesion, growth, biofilm formation, and EAP1, HWP1 and SAP2 gene expression. BMC Microbiol. 2014;14:61.

41. Yeater KM, Chandra J, Cheng G, et al. Temporal analysis of Candida albicans gene expression during biofilm development. Microbiology. 2007;153:2373-85.

42. Samaranayake YH, Cheung BPK, Yau JYY, et al. Human serum promotes Candida albicans biofilm growth and virulence gene expression on silicone biomaterial. PLoS ONE. 2013;8:e62902.

43. Vilela SF, Barbosa JO, Rossoni RD, et al. Lactobacillus acidophilus ATCC 4356 inhibits biofilm formation by $C$. albicans and attenuates the experimental candidiasis in Galleria mellonella. Virulence. 2015;6:29-39.

44. Rossoni RD, Barbosa JO, Vilela SFG, et al. Competitive Interactions between C. albicans, C. glabrata and C. krusei during biofilm formation and development of experimental Candidiasis. PLoS ONE. 2015;10:e0131700.

45. Bergin D, Brennan M, Kavanagh K. Fluctuations in haemocyte density and microbial load may be used as indicators of fungal pathogenicity in larvae of Galleria mellonella. Microbes Infect. 2003;5:1389-95. 